\title{
APPLICATION OF THE CHROMOSPHERIC MAGNETOGRAPH TO ACTIVE REGIONS
}

\author{
H. ZIRIN \\ Hale Observatories, Carnegie Institution of Washington, \\ California Institute of Technology, Pasadena, Calif., U.S.A.
}

\begin{abstract}
We show how to determine the magnetic field structure in active regions from the $\mathrm{H} \alpha$ morphology. We also show the role of the EFR (emerging flux region) as a bipolar region of velocity downflow. Finally, we point out that since all new magnetic flux emerges in strictly bipolar form, complex spot groups must result from surface interaction, hence most of the solar surface field may be produced on the surface.
\end{abstract}

\section{Introduction}

In two previous papers (Veeder and Zirin, 1970; Zirin, 1970) we have shown how chromospheric morphology may be used to deduce magnetic field structure. The bases of this program are the facts that any field strong enough to be of interest should produce tangible effects in the chromosphere, and that I am too lazy to build one more magnetograph. Unfortunately, our understanding of the Sun is not so far advanced that we can predict the relation between field and morphology, so we must find a Rosetta stone in the form of a high quality pair of magnetogram and filtergram. This problem is particularly difficult in active regions, where field patterns are particularly complex.

In the present paper I present new field-morphology correspondence drawn from 'fine scan' magnetograms made at Mt. Wilson and high resolution filtergrams made at Big Bear. From two such sets we draw the following rules, which should be added to those of our preceding papers:

(1) Every magnetic structure on the fine scan magnetogram appears as a chromospheric feature of some sort.

(2) Field transitions are marked by brightness transitions or by dark regions.

(3) The plage-antiplage discrepancy found by Veeder and Zirin is not correct; preceding plage is as bright or brighter than following plage; but $p$ spots often have extensive regions of horizontal field surrounding them, which are the 'antiplage'.

(4) Emerging flux regions (EFR) are marked on magnetograms by bipolar magnetic symmetry and velocity downflow.

\section{October 25, 1969}

Our first exhibit is an $\mathrm{H} \alpha$-magnetogram pair made October 25,1969 ; the magnetogram (Figure 2) was made by Dr. A. Bhatnagar at Mt. Wilson. The magnetogram must be rotated somewhat to conform to the $\mathrm{H} \alpha$ picture, but we see that each fine detail appears on both pictures. I have marked salient regions on Figures 1 and 2 . We see 


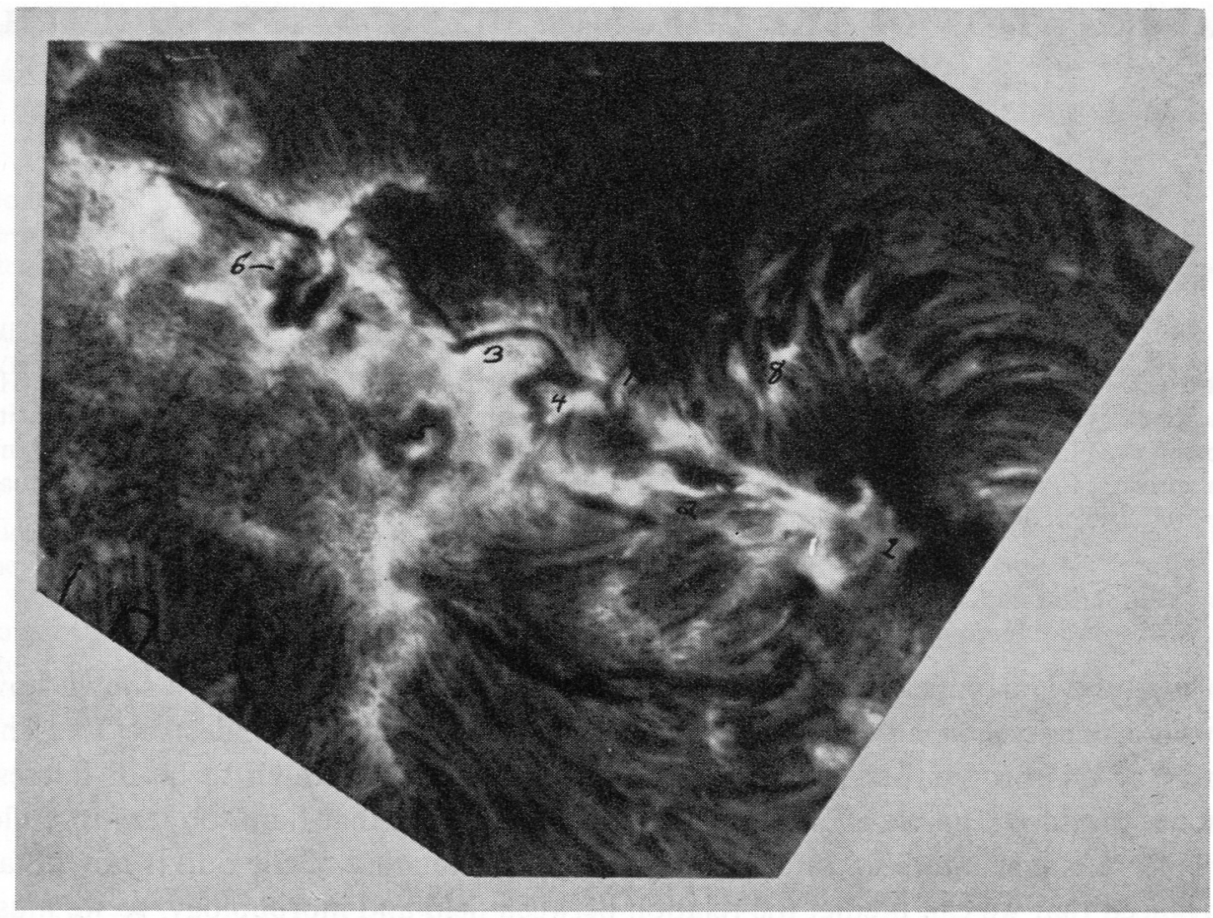

Fig. 1. H $\alpha$ picture made October 25, 1969.

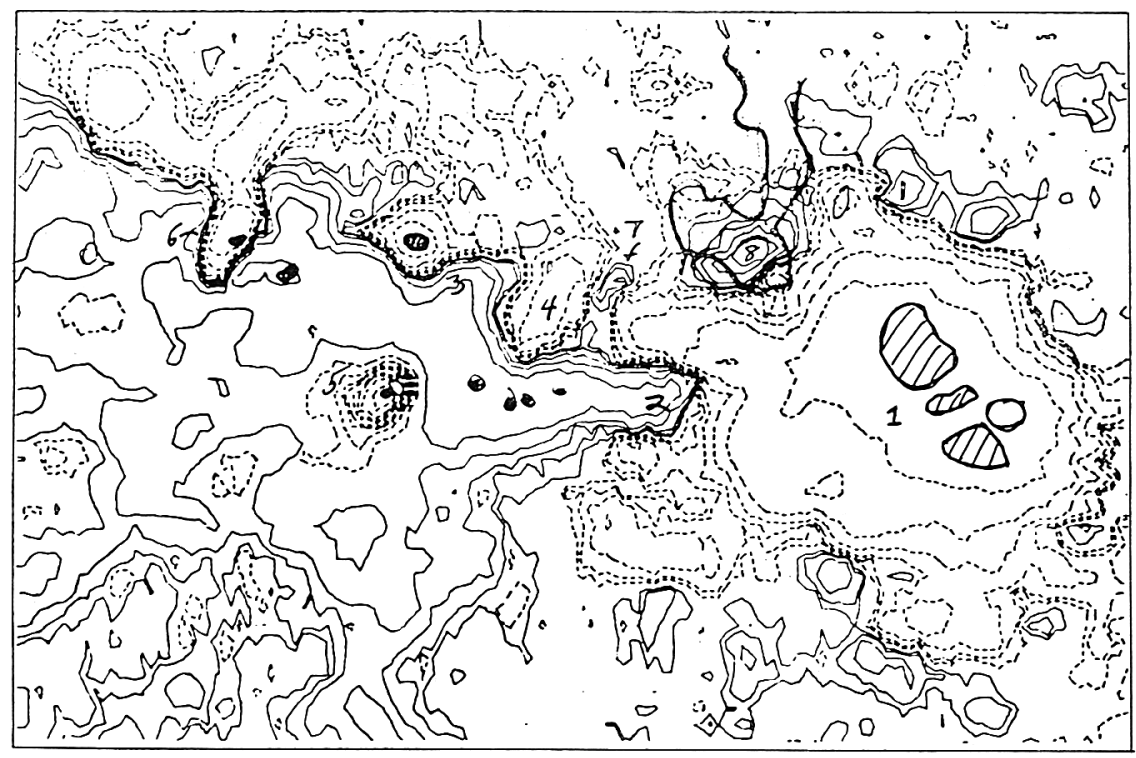

Fig. 2. Magnetogram made by Dr. A. Bhatnagar at Mt. Wilson Observatory October 25, 1969. 
a large, complex group, dominated by preceding spots (1). The boundary to following polarity is not well marked but may be distinguished at (2). From this point to the left we see a ragged division line running NE-SW, dividing the following plage from an antiplage of preceding polarity; the dividing line is clearly marked by the filaments (3). Note the inclusions of preceding polarity (4) and (5). These are seen in white light to have small spots, and are clearly defined as polarity changes by the dark radial $\mathrm{H} \alpha$ structure surrounding their bright centers. The inclusion (6) is bipolar and inverted; a small following spot leads the preceding spot in (6); considerable flare activity occurred in this region.

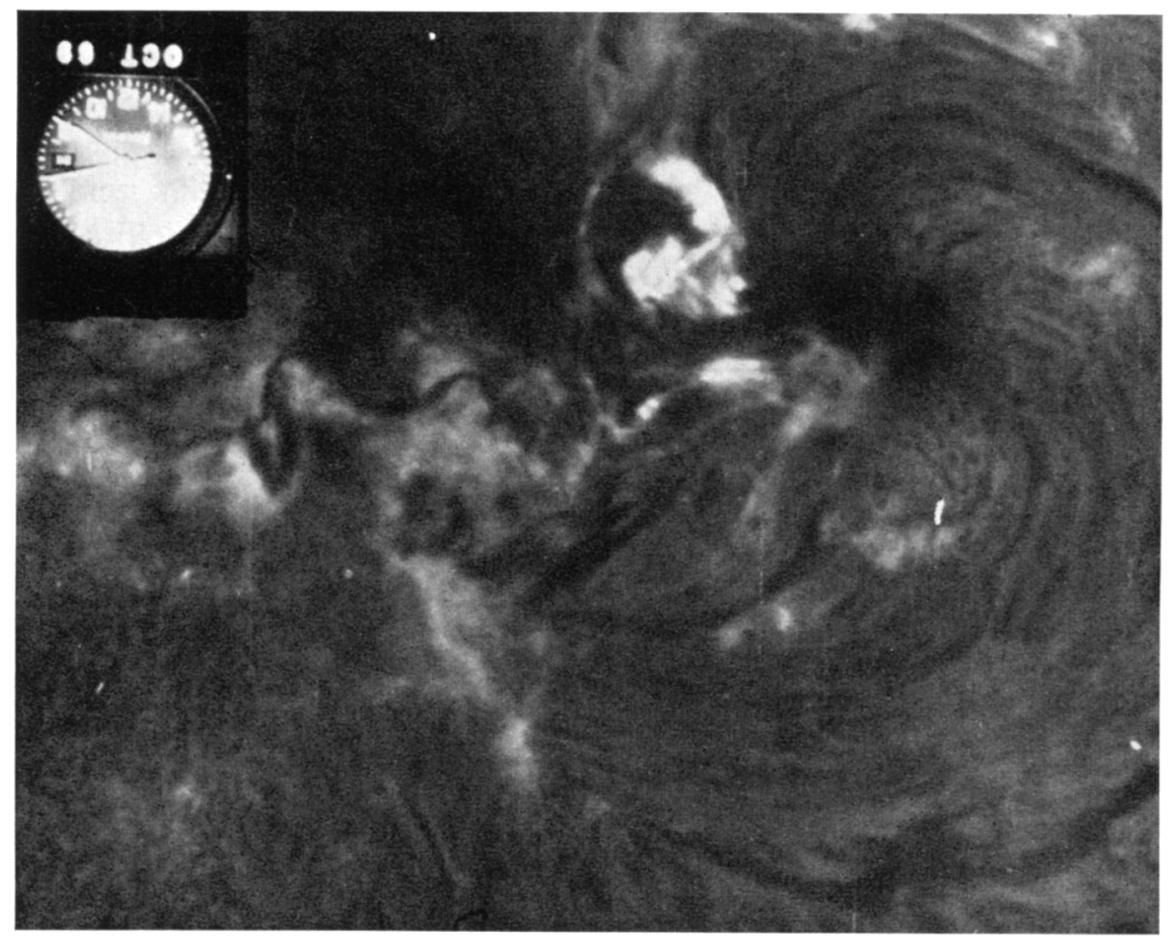

Fig. 3. Picture of following polarity.

Regions 7 and 8 are included areas of following polarity; 7 is particularly well marked by a dark boundary. A sizeable flare occurred here on the 24 th; in the picture (Figure 3) we see how closely it sticks to the region of following polarity. In summary, we see that the $\mathrm{H} \alpha$ morphology in this group corresponds strictly to the magnetic field, and given adequate optical resolution, one can normally draw a magnetogram from the $H \alpha$ pictures.

\section{April 10-11, 1970}

The second comparison set was made April 10 and 11, 1970; fine scan magnetograms 

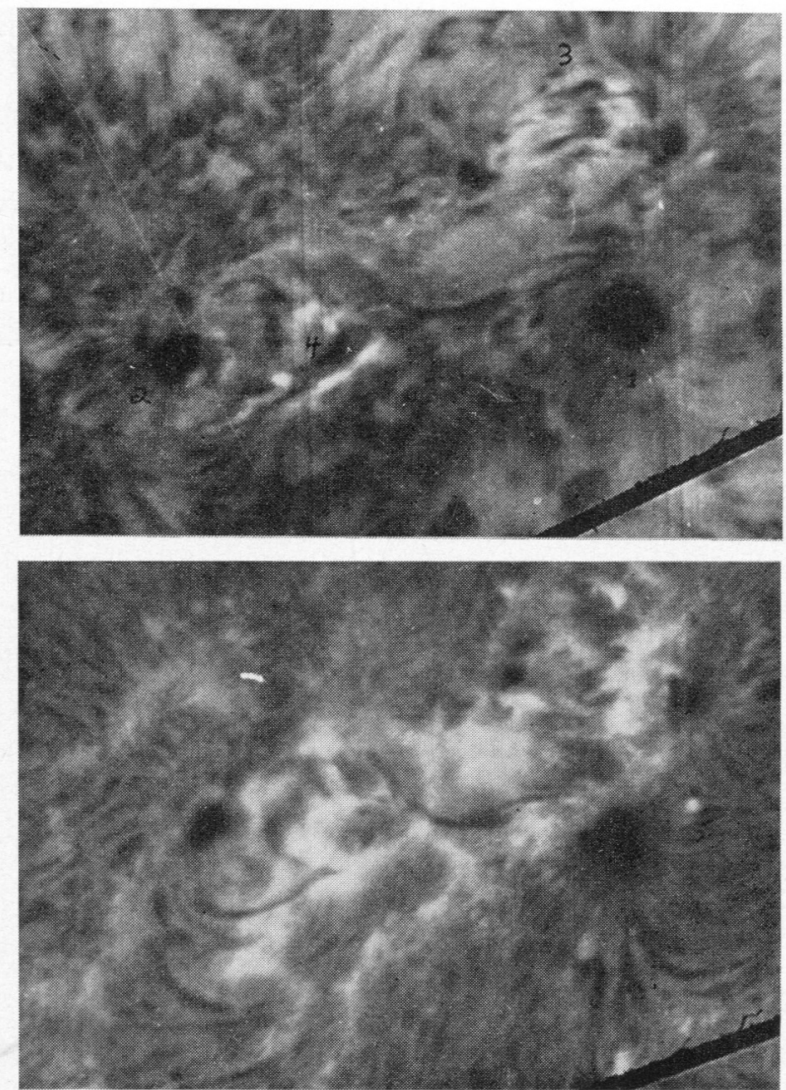

Fig. 4. Big Bear off-band pictures showing emerging flux region. Top: April 10, 1970. Bottom: April 11, 1970.

B
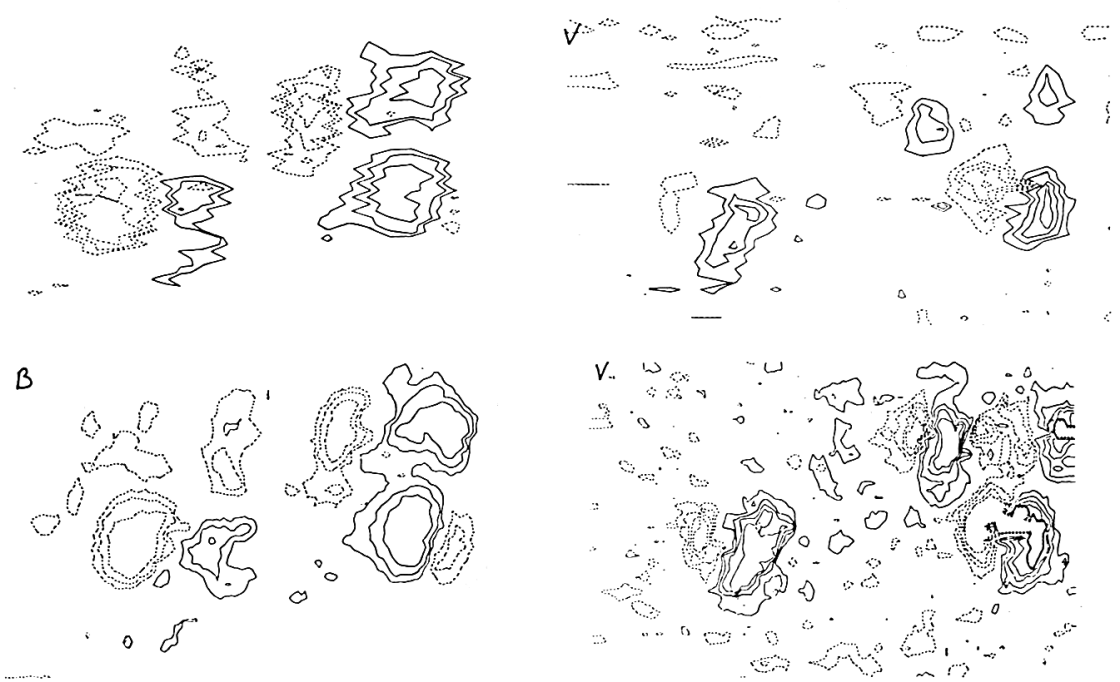

Fig. 5. Magnetograms showing emerging flux region. 
were obtained by John Adkins. The resolution of the magnetograms was only $5 \times 25 \mathrm{~s}$ on the 10th, but was 5 arc sec square on the 11 th.

We see in Figure 4a mature bipolar spot group (McMath plage 10669) with large preceding (1) and following (2) spots. The field distribution varies from that common in bipolar spots because this is an older group, several rotations old, which is primarily divided by the filament connecting the two spots into a following plage above and preceding polarity below. At 1622 on April 10 there was a large flare in two parts of the preceding region, the first part occurring behind the preceding spot, followed by brightening in the area (4) just ahead of the following spot. Figure 4 shows Big Bear off-band pictures; we also have smaller scale centerline $\mathrm{H} \alpha$ pictures from Pasadena. Unfortunately, the sensitivity of magnetogram (Figure 5) is low so that it does not show the weaker plage fields detectable in $\mathrm{H} \alpha$; however, since most people are still more willing to believe the Babcock magnetogram, no matter how poor, before the chromospheric magnetogram, we make the instructive comparisons.

The most interesting feature in Figures 4 and 5 is the presence of an emerging flux region (EFR) directly $\mathbf{N}$ of the spot group.

These have been referred to as AFS-arched filament systems by Bruzek, (1969) and as BRL-bright regions with loops by Weart and Zirin (1968), but it is now well established that they are emerging flux regions, and I shall use that term. The EFR are typically bipolar, and, as was established by Roberts (1969), they show downflow at either end. In Figure $4 b$ we see that the EFR matured the next day. We also see on this more sensitive velocity picture that there is an upflow in the center of the region. We also see a new region of following polarity (5) emerging ahead of the large spot. This never amounted to much.

I should like to point out an important feature of the EFR's. Flux invariably emerges in bipolar form, yet important spot groups such as that in Figure 1, almost never show this bipolar form. Very often we find an inverted polarity resulting from rapid growth of a bipolar EFR ahead of a preceding spot - the preceding part of the EFR fades and we are left with a strong polarity inversion. More often, we find an EW dividing line following big preceding spots, as in Figure 1. It would appear that the form of sunspot groups, as well as most of their flux, is the result of surface interactions which amplify and distort the new fields into large and complex spots of different orientations. It is perhaps significant that normal bipolar spot groups, which might be termed 'unevolved' are small and show little activity.

In summary, we have shown that further progress in the chromospheric magnetograph now permits the measurement of active region fields with reasonable accuracy. All field changes correspond to visible features in the chromosphere. The interpretation of these features is somewhat harder, but it seems clear that we see dark regions in $\mathrm{H} \alpha$ wherever the field is horizontal.

\section{Acknowledgements}

I am indebted to A. Bhatnagar and J. Adkins for magnetograms, and to Peter Foukal 
for numerous discussions. This work was supported by NASA and by the Atmospheric Sciences section of the NSF.

\section{References}

Bruzek, A.: 1967, in K. O. Kiepenheuer (ed.), 'Structure and Development of Solar Active Regions', IAU Symp. 35, 293.

Roberts, P. R.: 1969, Thesis, California Institute of Technology.

Veeder, G. and Zirin, H.: 1970, Solar Phys. 12, 391.

Weart. S. and Zirin, H.: 1969, Publ. Astron. Soc. Pacific, 81, 480.

Zirin, H.: 1970, Paper presented to Royal Society Special Discussion on Solar Studies. 